\title{
Energetic Chromophores: Low Energy Laser Initiation in Explosive Fe(II) Tetrazine Complexes
}

Thomas W. Myers ${ }^{1 *}$, Josiah A. Bjorgaard ${ }^{2}$, Kathryn E. Brown 3 , David E. Chavez', Susan K. Hanson $^{4}$, R. Jason Scharff', Sergei Tretiak², Jacqueline M. Veauthier ${ }^{4}$.

Contribution from M division'; Theoretical division ${ }^{2}$; W division 3 ; and Chemistry division ${ }^{4}$; Los Alamos National Laboratory, P. O. Box 1663, Los Alamos, NM 87545 (USA). 
Table S1. Crystallographic information for 11-13, and 15.

\begin{tabular}{|c|c|c|c|c|}
\hline & $\mathbf{1 1}$ & 12 & 13 & 15 \\
\hline Formula & $\mathrm{C}_{27} \mathrm{H}_{38} \mathrm{Cl}_{2} \mathrm{FeN}_{24} \mathrm{O}_{9}$ & $\mathrm{C}_{21} \mathrm{H}_{24} \mathrm{Cl}_{2} \mathrm{FeN}_{24} \mathrm{O}_{8}$ & $\mathrm{C}_{26} \mathrm{H}_{27} \mathrm{Cl}_{2} \mathrm{FeN}_{25} \mathrm{O}_{8}$ & $\mathrm{C}_{39} \mathrm{H}_{62} \mathrm{Cl}_{2} \mathrm{FeN}_{32} \mathrm{O}_{13}$ \\
\hline Crystal size & $0.14 \times 0.21 \times 0.25$ & $0.12 \times 0.21 \times 0.78$ & $0.13 \times 0.17 \times 0.21$ & $0.15 \times 0.23 \times 0.25$ \\
\hline Formula weight, $\mathrm{g} \mathrm{mol}^{-1}$ & 969.56 & 867.39 & 944.47 & 1313.96 \\
\hline Space group & $\mathrm{P}_{2} / \mathrm{c}$ & $\mathrm{P}_{2} / \mathrm{n}$ & $\mathrm{P}_{2} / \mathrm{n}$ & $\mathrm{P} \overline{1}$ \\
\hline$a, \AA$ & $19.10(5)$ & $21.211(4)$ & $11.605(14)$ & $12.941(10)$ \\
\hline$b, \AA$ & $13.96(4)$ & $8.664(2)$ & $16.245(18)$ & $15.790(12)$ \\
\hline$c, \AA$ & $16.03(6)$ & $21.347(4)$ & $20.204(19)$ & $16.280(13)$ \\
\hline$\alpha, \operatorname{deg}$ & 90 & 90 & 90 & $115.955(7)$ \\
\hline$\beta$, deg & $109.165(6)$ & $115 \cdot 966(2)$ & $97.184(7)$ & $100.085(7)$ \\
\hline$\gamma$, deg & 90 & 90 & 90 & $94.838(7)$ \\
\hline$V, \AA^{3}$ & $4039(2)$ & $3527.1(11)$ & $3778(9)$ & $2896(4)$ \\
\hline$Z$ & 4 & 4 & 4 & 2 \\
\hline$T, \mathrm{~K}$ & $100(2)$ & $100(2)$ & $100(2)$ & $100(2)$ \\
\hline$P$, calcd, $\mathrm{g} \mathrm{cm}^{-3}$ & 1.595 & 1.633 & 1.661 & 1.507 \\
\hline Refl. collected $/ 2 \theta_{\max }$ & $36544 / 56.42$ & $36630 / 53.15$ & $35945 / 52.89$ & $22314 / 52.20$ \\
\hline Unique refl./ I>2 $\sigma(\mathrm{I})$ & $9626 / 7148$ & $7346 / 6155$ & $7742 / 5460$ & $11163 / 4889$ \\
\hline Parameters/restraints & $576 / 0$ & $525 / 8$ & $578 / 0$ & $784 / 0$ \\
\hline$\lambda, \AA / \mu(\mathrm{K} \alpha), \mathrm{cm}^{-1}$ & 0.71073 & 0.71073 & 0.71073 & 0.71073 \\
\hline $\mathrm{R}_{1} / \mathrm{GOF}$ & $0.0438 / 1.050$ & $0.0515 / 1.116$ & $0.0460 / 1.024$ & $0.0835 / 0.990$ \\
\hline$w R_{2}(I>2 \sigma(I))$ & 0.1017 & 0.1324 & 0.0993 & 0.2004 \\
\hline Residual density, e $\AA^{-3}$ & $0.820 /-0.695$ & $0.981 /-0.803$ & $0.418 /-0.814$ & $0.759 /-0.770$ \\
\hline
\end{tabular}

Table S2. Selected average bond lengths and angles in 11-13, and 15.

\begin{tabular}{|c|c|c|c|c|}
\hline & 11 & 12 & 13 & 15 \\
\hline $\mathrm{Fe}-\mathrm{N}_{\mathrm{Tz}}$ & $1.907(6)$ & $1.895(2)$ & $1.911(3)$ & $1.894(5)$ \\
\hline Fe- $N_{\mathrm{Pyr}}$ & $1.953(6)$ & $1.978(2)$ & $1.980(3)$ & $1.959(5)$ \\
\hline $\mathrm{N}_{\mathrm{pyr}}-\mathrm{N}_{\mathrm{Pyr}}$ & $1.365(4)$ & $1.378(3)$ & 1.393(3) & $1.388(5)$ \\
\hline $\mathrm{N}_{\mathrm{Pyr}}-\mathrm{C}_{\mathrm{Tz}}$ & $1.388(4)$ & $1.385(3)$ & $1.387(3)$ & $1.383(5)$ \\
\hline $\mathrm{C}_{\mathrm{Tz}}-\mathrm{N}_{\mathrm{Tz}}(1)$ & $1.343(4)$ & $1.345(3)$ & $1.402(3)$ & $1.388(5)$ \\
\hline $\mathrm{C}_{\mathrm{Tz}}-\mathrm{N}_{\mathrm{Tz}}(2)$ & $1.354(4)$ & $1.355(3)$ & $1.348(3)$ & $1.358(5)$ \\
\hline $\mathrm{C}_{\mathrm{Tz}}-\mathrm{N}_{\mathrm{Tz}}(3)$ & $1.363(4)$ & $1.359(3)$ & $1.379(3)$ & $1.383(5)$ \\
\hline $\mathrm{C}_{\mathrm{Tz}}-\mathrm{N}_{\mathrm{Tz}}(4)$ & $1.319(4)$ & $1.314(3)$ & $1.299(3)$ & $1.311(5)$ \\
\hline $\mathrm{N}_{\mathrm{Tz}}-\mathrm{N}_{\mathrm{Tz}}(1)$ & $1.318(4)$ & $1.314(3)$ & $1.307(3)$ & $1.311(5)$ \\
\hline $\mathrm{N}_{\mathrm{Tz}}-\mathrm{N}_{\mathrm{Tz}}(2)$ & $1.320(4)$ & $1.318(3)$ & $1.358(3)$ & $1.358(5)$ \\
\hline $\mathrm{NH}_{2}-\mathrm{C}_{\mathrm{Tz}}$ & $1.328(4)$ & $1.317(3)$ & N/A & N/A \\
\hline $\mathrm{C}_{\mathrm{Tz}}-\mathrm{N}_{\mathrm{Tri}}$ & $\mathrm{N} / \mathrm{A}$ & N/A & $1.333(3)$ & $1.313(5)$ \\
\hline $\mathrm{N}_{\text {Tri }}-\mathrm{N}_{\text {Tri }}$ & $\mathrm{N} / \mathrm{A}$ & $\mathrm{N} / \mathrm{A}$ & $1.366(3)$ & $1.357(5)$ \\
\hline $\mathrm{N}_{\text {Tri }}-\mathrm{C}_{\mathrm{Tri}}$ & $\mathrm{N} / \mathrm{A}$ & $\mathrm{N} / \mathrm{A}$ & $1.331(3)$ & $1.340(5)$ \\
\hline $\mathrm{C}_{\mathrm{Tri}}-\mathrm{NH}_{2}$ & $\mathrm{~N} / \mathrm{A}$ & $\mathrm{N} / \mathrm{A}$ & $\mathrm{N} / \mathrm{A}$ & $1.321(5)$ \\
\hline $\mathrm{C}_{\mathrm{Tri}}-\mathrm{N}_{\mathrm{Tz}}$ & $\mathrm{N} / \mathrm{A}$ & $\mathrm{N} / \mathrm{A}$ & $1.342(3)$ & $1.332(5)$ \\
\hline $\mathrm{N}_{\mathrm{pyr}}-\mathrm{Fe}-\mathrm{N}_{\mathrm{Tz}}$ & $81.63(3)$ & $81.01(10)$ & $81.63(11)$ & $81.3(2)$ \\
\hline $\mathrm{N}_{\mathrm{Tz}}-\mathrm{Fe}-\mathrm{N}_{\mathrm{Tz}}$ & $93 \cdot 30(4)$ & $92.82(10)$ & $92.13(12)$ & $92.5(2)$ \\
\hline $\mathrm{N}_{\mathrm{pyr}}-\mathrm{Fe}-\mathrm{N}_{\mathrm{Pyr}}$ & $93 \cdot 52(4)$ & $96.32(10)$ & $96.80(12)$ & $97.0(2)$ \\
\hline $\mathrm{N}_{\mathrm{Tz}}-\mathrm{Fe}-\mathrm{N}_{\mathrm{Pyr}}($ trans $)$ & $172.90(8)$ & $173 \cdot 31(14)$ & $173.47(17)$ & $173.6(2)$ \\
\hline $\mathrm{N}_{\mathrm{Tz}}-\mathrm{Fe}-\mathrm{N}_{\mathrm{Pyr}}($ cis $)$ & $91.99(3)$ & $90.07(10)$ & $89.63(11)$ & $89.3(2)$ \\
\hline
\end{tabular}


Table S3. Comparison of simulated and experimental $\mathrm{Fe}-\mathrm{N}_{\mathrm{Pyr}}$ and $\mathrm{Fe}-\mathrm{N}_{\mathrm{Tz}}$ bond lengths for optized geometries in vacuum and in solution.

\begin{tabular}{|c|c|c|c|c|c|c|}
\hline \multirow{2}{*}{} & \multicolumn{3}{|c|}{ Fe- $\mathrm{N}_{\mathrm{Pyr}}$ Bond length (A) } & \multicolumn{3}{c|}{ Fe- $\mathrm{N}_{\mathrm{Tz}}$ Bond lengths (A) } \\
\cline { 3 - 4 } & \multirow{2}{*}{ Experiment } & \multicolumn{2}{|c|}{ Theory } & \multirow{2}{*}{ Experiment } & \multicolumn{2}{c|}{ Theory } \\
\cline { 3 - 4 } & & Gas Phase & Solution & & Gas Phase & Solution \\
\hline 11 & $1.953(6)$ & 2.03 & 2.03 & $1.907(6)$ & 1.96 & 1.95 \\
\hline 12 & $1.978(2)$ & 2.00 & 2.00 & $1.895(2)$ & 1.98 & 1.96 \\
\hline 13 & $1.980(3)$ & 2.04 & 2.02 & $1.911(3)$ & 1.96 & 1.95 \\
\hline 14 & - & 2.00 & 1.99 & - & 1.97 & 1.97 \\
\hline 15 & $1.959(5)$ & 2.04 & 2.02 & $1.894(5)$ & 1.96 & 1.96 \\
\hline 16 & - & 2.00 & 1.99 & - & 1.98 & 1.97 \\
\hline
\end{tabular}

Table S4. Calculated total energies in Hartrees.

\begin{tabular}{|c|c|c|c|}
\hline & $E(+1)$ (a.u.) & $E(-1)$ (a.u.) & $E($ o $)$ (a.u.) \\
\hline $\mathbf{1 1}$ & 1853.14490 & $\mathrm{o}$ & -1853.38397 \\
\hline $\mathbf{1 2}$ & -2089.07495 & 0 & -2089.30519 \\
\hline $\mathbf{1 3}$ & -2129.75477 & -2130.17712 & -2130.00391 \\
\hline $\mathbf{1 4}$ & -2365.68718 & -2366.09769 & -2365.92828 \\
\hline $\mathbf{1 5}$ & -2295.88031 & -2296.27013 & -2296.10940 \\
\hline $\mathbf{1 6}$ & -2531.80951 & -2532.18962 & -2532.03186 \\
\hline
\end{tabular}


Hole 2 Hole $1 \quad$ Elec. $1 \quad$ Elec. 2

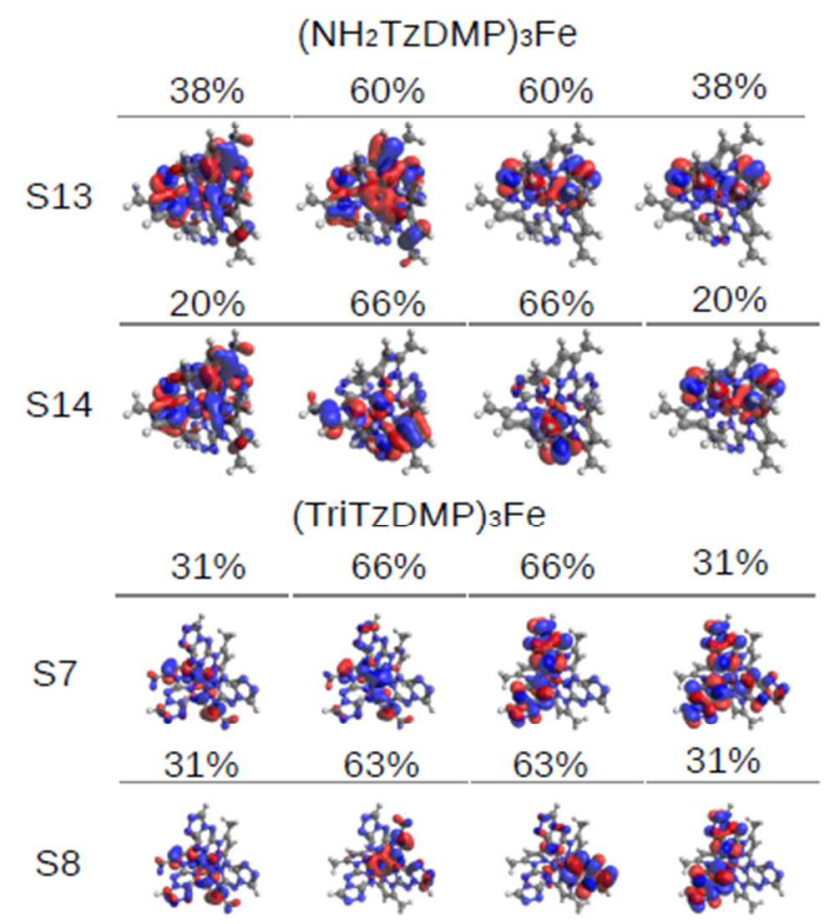

Figure S1. The dominant natural transition orbital pairs for the first two excited states $\left(\mathrm{S}_{13}, \mathrm{~S}_{14}\right)$ of 11, and the first two excited states $\left(\mathrm{S}_{7}, \mathrm{~S} 8\right)$ of $\mathbf{1 3}$. Contributing holes for each state on the left and contributing particles on the right.

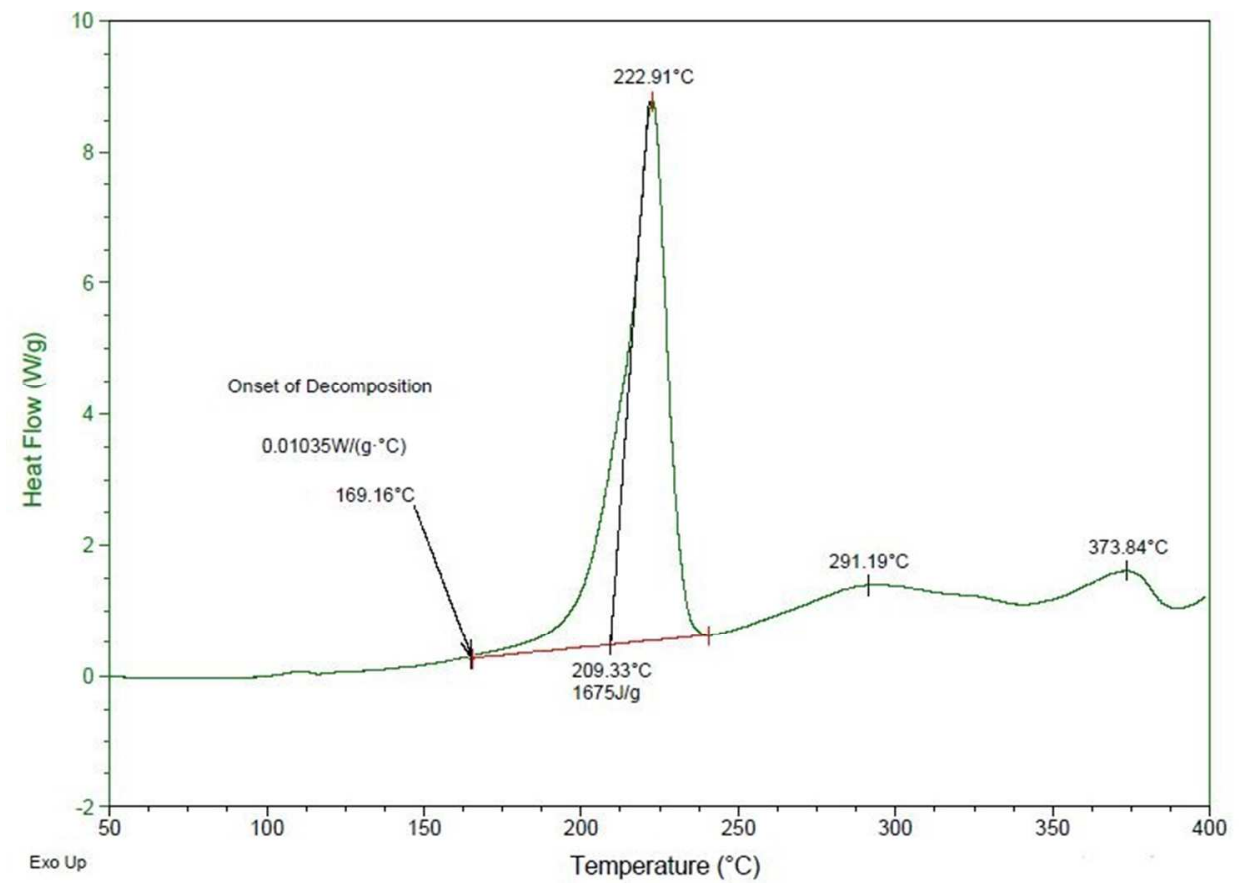

Figure S2. DSC plot of 11 at a heating rate of $10{ }^{\circ} \mathrm{C} \min ^{-1}$ (Exo up). 


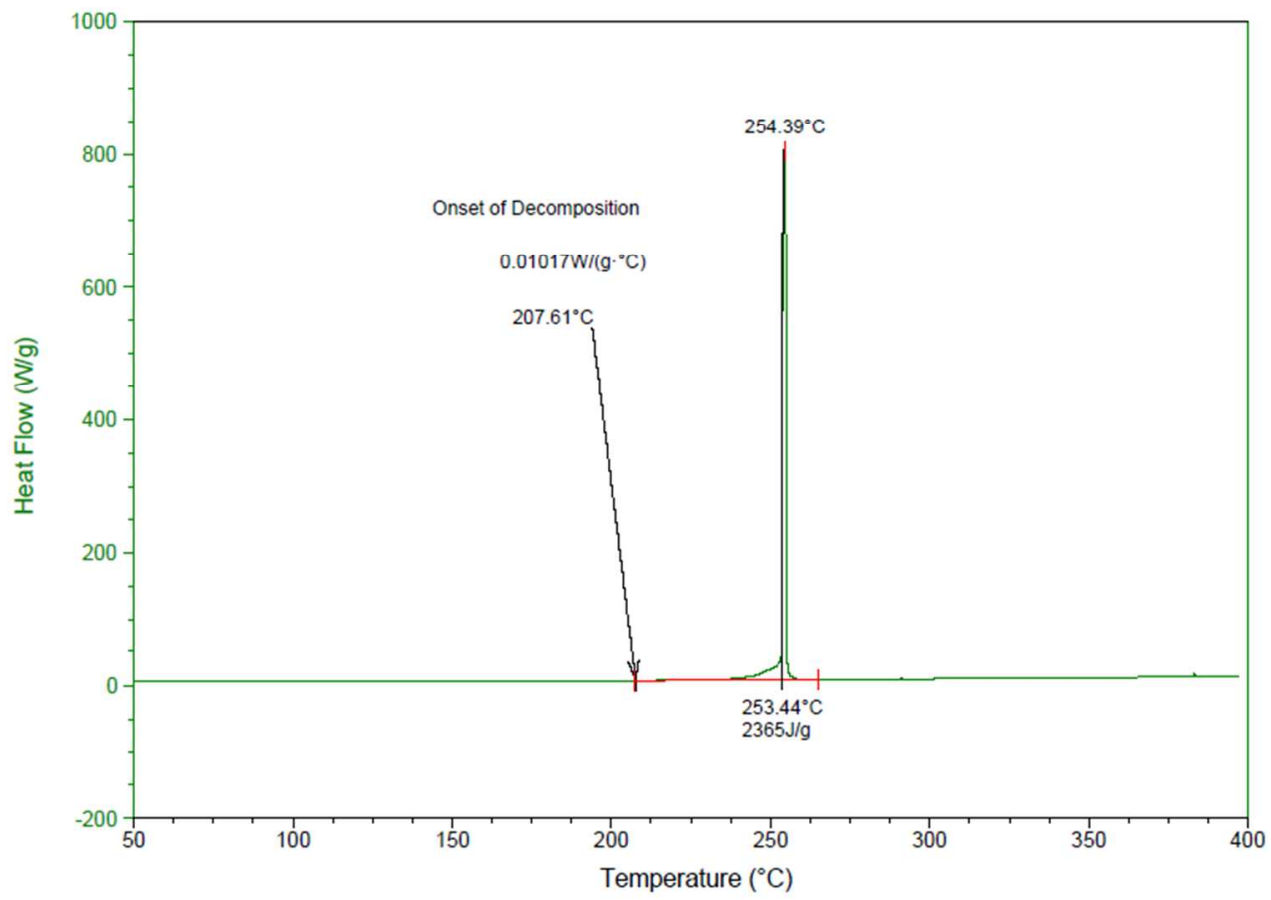

Figure S3. DSC plot of 12 at a heating rate of $10^{\circ} \mathrm{C} \min ^{-1}$ (Exo up).

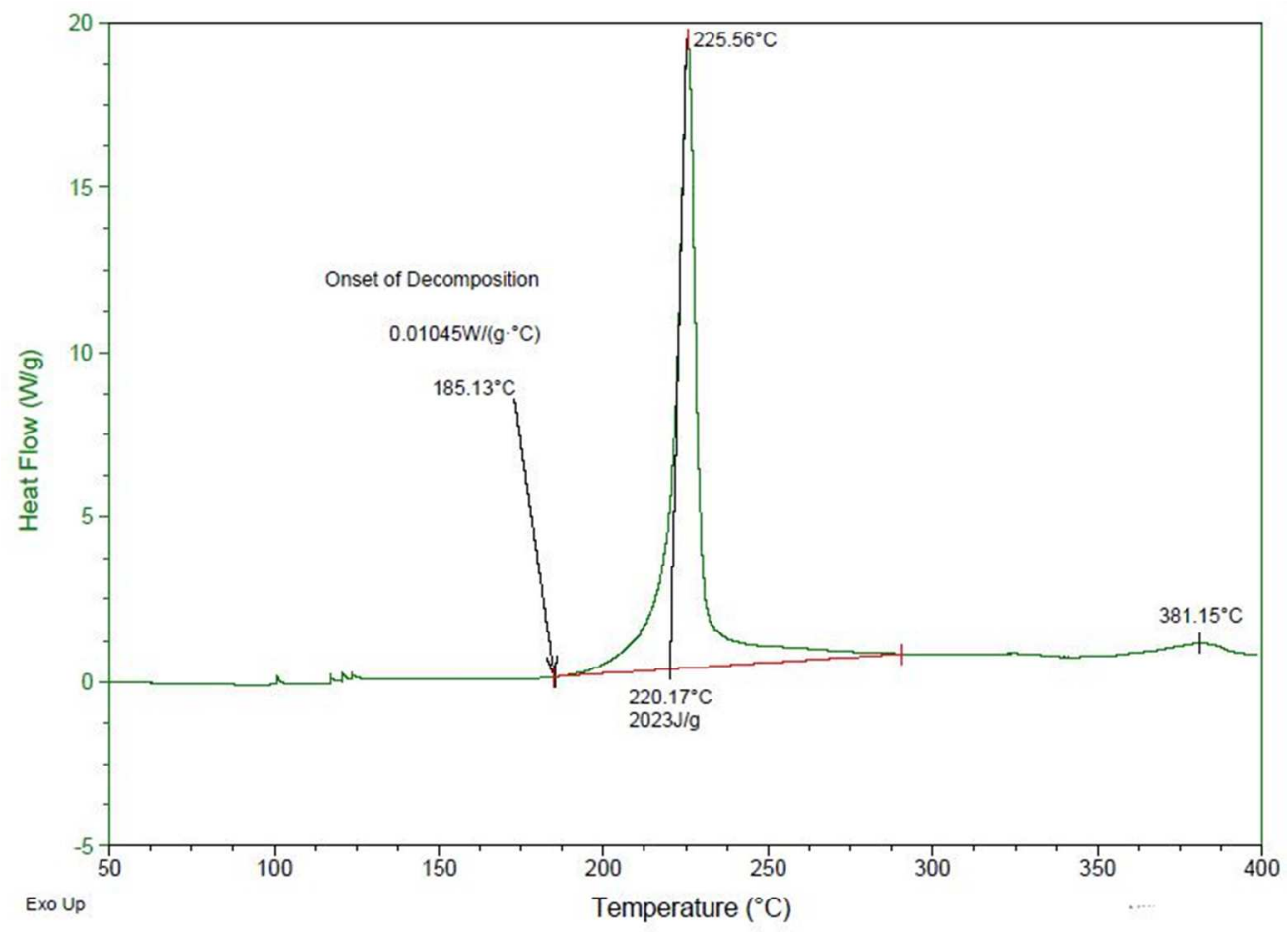

Figure $\mathrm{S}_{4}$. DSC plot of 13 at a heating rate of $10{ }^{\circ} \mathrm{C} \mathrm{min}^{-1}$ (Exo up). 


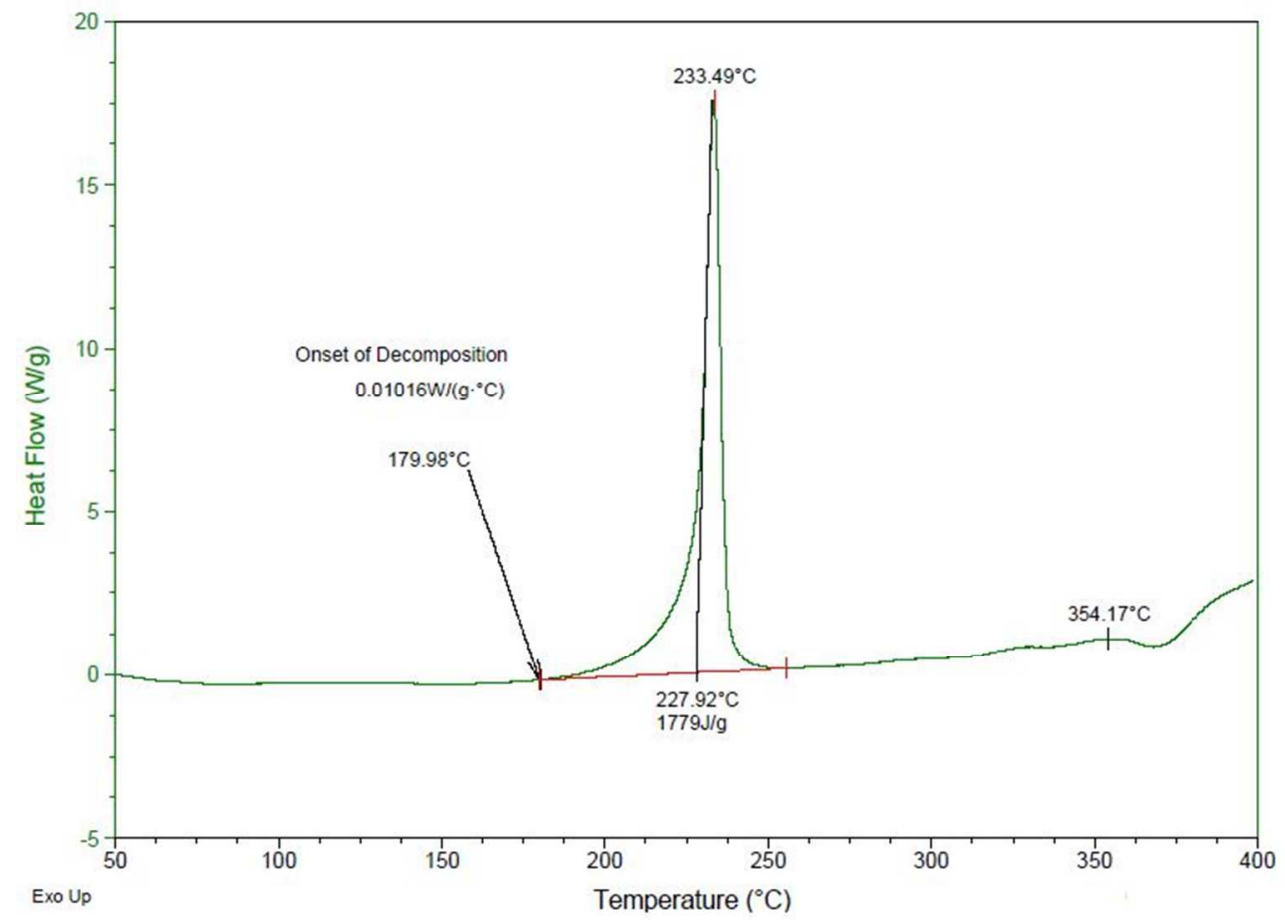

Figure $\mathrm{S}_{5}$. DSC plot of 14 at a heating rate of $10^{\circ} \mathrm{C} \mathrm{min}^{-1}$ (Exo up).

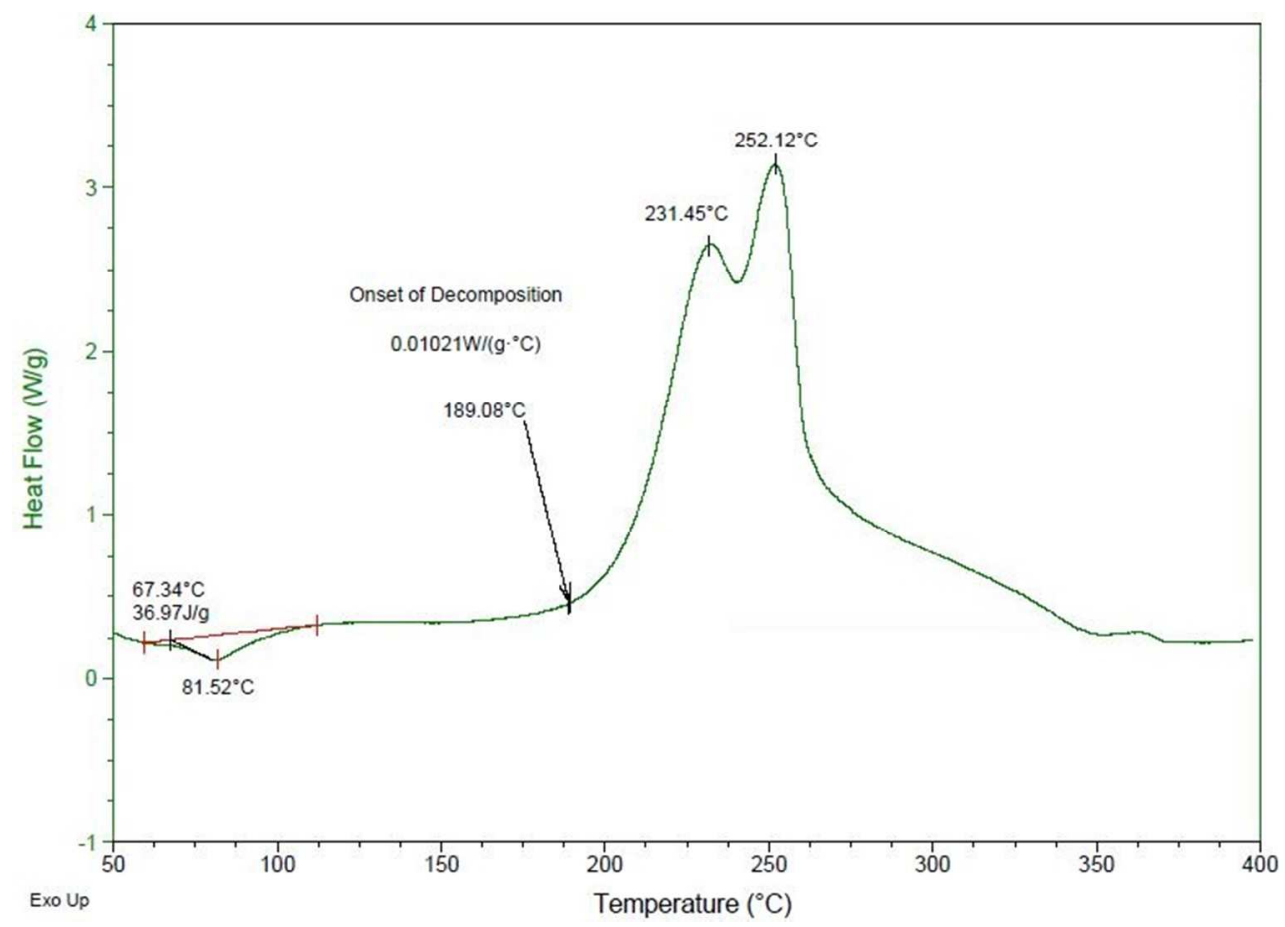

Figure S6. DSC plot of 15 at a heating rate of $10{ }^{\circ} \mathrm{C} \min ^{-1}$ (Exo up). 


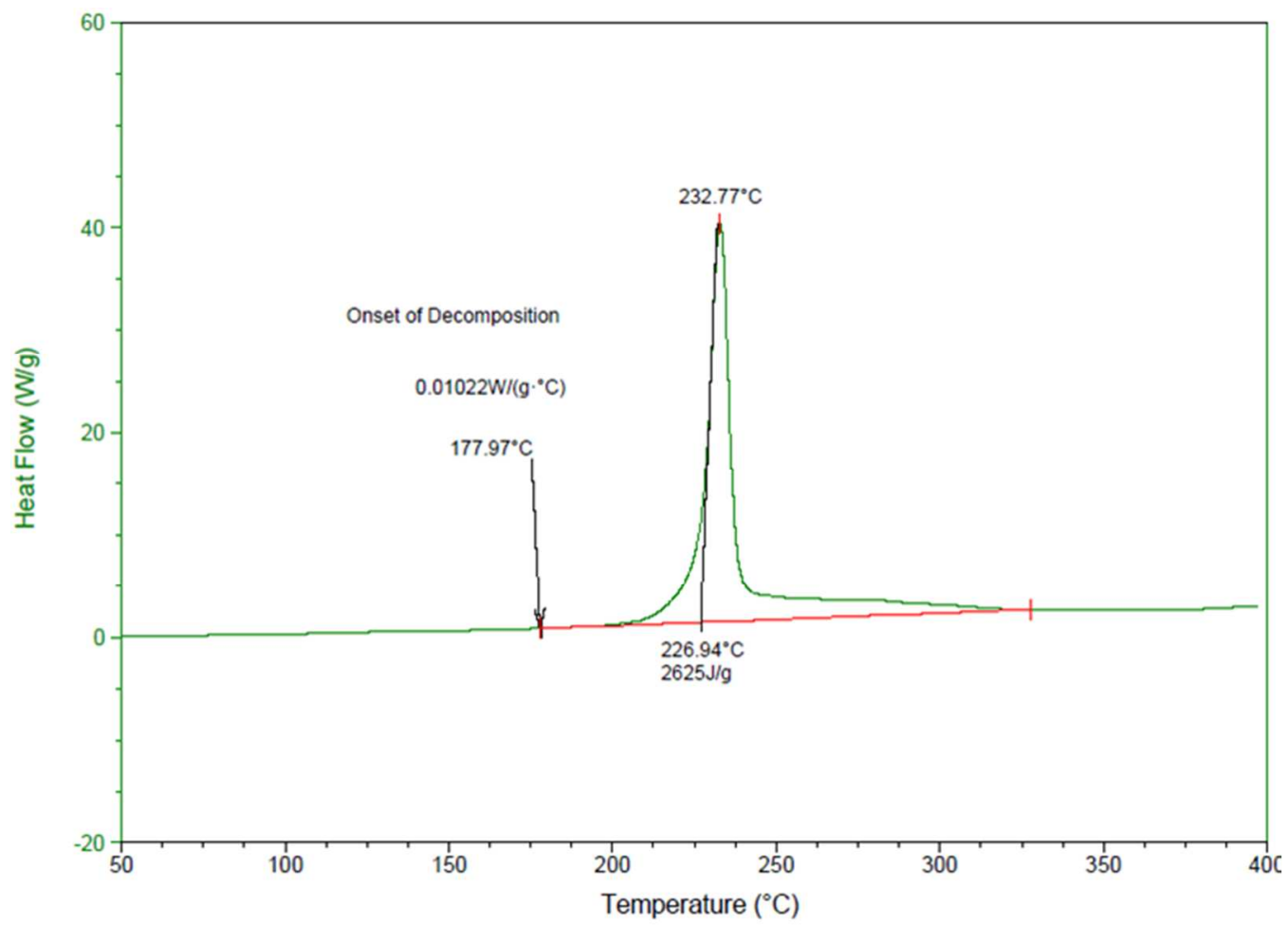

Figure S7. DSC plot of 16 at a heating rate of $10{ }^{\circ} \mathrm{C} \min ^{-1}$ (Exo up). 